\title{
El perfil de los responsables de comunicación interna en España
} The profile of internal communication managers in Spain

\section{Susana Miquel Segarra}

Universitat Jaume I (UJI)

smiquel@uji.es

Cristina Aced

Universitat Oberta de Catalunya (UOC)

caced@uoc.edu

Forma de citar este artículo:

Miquel Segarra, S. y Aced, C. (2019). "El perfil de los responsables de comunicación interna en España", RAEIC, Revista de la Asociación Española de Investigación de la Comunicación, vol 6, núm. 11, 99-118.

DOI: https://doi.org/10.24137/raeic.6.11.7

\section{Resumen:}

Una condición imprescindible para que la comunicación interna $(\mathrm{Cl})$ sea eficaz es la implicación de la gerencia y los altos cargos. Estudios recientes realizados a escala internacional confirman que los líderes empresariales están prestando más atención que nunca a la comunicación interna. Esta circunstancia hace que la comunicación interna esté creciendo con rapidez a escala internacional y en España. Por ello resulta conveniente conocer el perfil de los profesionales que están asumiendo estas tareas para constatar que se adecúan a las funciones propias del puesto que desempeñan. Así pues, el presente trabajo analiza las características sociolaborales de los profesionales 
que se encargan de la comunicación interna en España, las estructuras en las que se integran y el posicionamiento de los departamentos, y su relación con otros departamentos de la empresa. Los primeros resultados revelan que los profesionales de $\mathrm{Cl}$ en España tienen un nivel académico muy elevado y se organizan en las empresas en equipos. Además, aunque todavía no cuentan con los condicionantes necesarios para tener una identidad propia, progresivamente están alcanzando mayor autonomía en las estructuras de las organizaciones.

Palabras clave: comunicación interna, perfil profesional, competencias, habilidades.

\section{Abstract:}

An essential condition for internal communication (IC) to be effective is the commitment of top executives and CEOs. Recent international studies confirm that business leaders are paying more attention than ever to internal communication. This circumstance means that internal communication is growing quickly on an international scale and also in Spain. For this reason, it is advisable to know the profile of the professionals who are in charge of these tasks with the aim to verify if they are adapted to the functions of their position. Thus, this work analyzes the social and working characteristics of professionals who are responsible for internal communication in Spain, the structures in which they are integrated, the location of the departments, and their relationship with other departments of the company. The first results reveal that IC professionals in Spain have a very high academic level and are organized in teams. In addition, although they still do not have the necessary conditions to have their own identity, progressively they are reaching greater autonomy in the structures of the organizations.

Keywords: internal communication, professional profile, skills, abilities.

\section{INTRODUCCIÓN}

Una condición imprescindible para que la comunicación interna $(\mathrm{Cl})$ sea eficaz es que la gerencia y los altos cargos se impliquen en su desarrollo (Cuenca y Verazzi, 2018; Tkalac Verčič y Pološki Vokić, 2017; Walden, Jung y Westerman, 2017; Ingelmo et al., 2018). Así, en línea con estudios anteriores (Grunig, 1992; Dozier y Broom, 1995; Ruck y 
Trainor, 2012), investigaciones más recientes ponen en evidencia que es difícil que la participación de los empleados mejore si los gerentes no se conciencian de la importancia de la comunicación interna. En la actualidad diversas investigaciones realizadas a escala internacional indican que los líderes empresariales están prestando cada vez más atención a la comunicación interna, y que en los próximos años esta tendencia irá en aumento (IC Kollectif, 2018).

A escala nacional la comunicación interna también se sitúa entre los principales objetivos de los departamentos de comunicación para los próximos tres años (Dircom, 2018). Sin embargo, todavía existe la percepción de que los directores de comunicación subestiman la $\mathrm{Cl}$ frente a otras disciplinas comunicativas desarrolladas en las organizaciones (VMA Group, 2018).

De hecho, aunque cada vez es más habitual que la $\mathrm{Cl}$ se integre en el departamento de comunicación, todavía hay muchas empresas en las que depende de otros equipos, como el de recursos humanos (RRHH) o el de marketing en menor medida (Cuenca y Verazzi, 2018). Pese a que no existe consenso sobre dónde debería ubicarse la $\mathrm{Cl}$, la mayoría de autores coincide en la importancia de que se cree un clima de colaboración entre el equipo de $\mathrm{Cl}$ y el resto de departamentos de la organización, en especial con el de RRHH (Corporate Excellence - Centre for Reputation Leadership, 2018; Xifra, 2014).

La creciente influencia de los responsables de $\mathrm{Cl}$ en las organizaciones se puede observar «cuando son invitados a reuniones de alto nivel que tratan sobre planificación estratégica a nivel organizativo. En este último caso, los comunicadores no solo son consultores internos, sino que forman parte del proceso de toma de decisiones» (Verčič, Zerfass, 2016, p. 276).

En esta línea, los informes European Communication Monitor (ECM) (2018), Internal Communication in Europe: Key success factors and managerial, approaches (2018) y The estate of the sector (Gatehouse, 2018) señalan que entre el 70 y el $76 \%$ de los encuestados afirma que el CEO o líder de su organización entiende el valor tanto de la comunicación corporativa y las relaciones públicas en general como de la comunicación interna en particular. Sin embargo, otras investigaciones de ámbito europeo indican que 
la influencia de estos profesionales es limitada: "Solo el $40 \%$ de los encuestados cree que la alta gerencia considera al equipo de IC como asesores de confianza» (Internal Communicaction in Europe, 2018). Así, tal y como revela el European Internal Communications (2018), parece que el valor de la Cl sigue siendo una preocupación en las organizaciones.

En este sentido, los encuestados para la redacción del informe The View (VMA Group, 2018) apuntan que los mayores desafíos para la profesión de la comunicación en los próximos cinco años son mejorar la reputación de la $\mathrm{Cl}$, justificar su papel y demostrar su eficacia. Por su parte, el European Communication Monitor (2018) también comparte entre las cuestiones estratégicas más importantes para la gestión de la comunicación hasta 2021 fortalecer el papel de la $\mathrm{Cl}$ para respaldar la toma de decisiones de la alta dirección. Y es que, a pesar de que la alta dirección comienza a reconocer la importancia de la $\mathrm{Cl}$ para su organización, no se sabe qué rol deben desempeñar sus profesionales, y con frecuencia los considera únicamente «técnicos de canales» (Volk el al., 2017). Esta incertidumbre también se pone de manifiesto en el estudio Making it Count, elaborado por CIPR Inside (2017). En él se afirma que los CEOs encuestados dicen que la $\mathrm{Cl}$ es una función estratégica, aunque ponen como ejemplos tareas que se refieren principalmente a cuestiones tácticas.

Sin embargo, para que una organización desarrolle una buena $\mathrm{Cl}$ su equipo de comunicación debe desarrollar competencias que van más allá de los aspectos tácticos (Vercic y Zerfass, 2016). Como señalan algunos autores (Tench et al., 2015, p. 42-44) estas competencias «son una combinación de conocimiento (lo que los profesionales deben saber para realizar su trabajo), habilidades (actividades que involucran ideas, personas o habilidades técnicas que los profesionales realizan con facilidad) y atributos personales (rasgos de carácter requeridos, actitudes y valores personales)». Dada la complejidad de estas habilidades profesionales es conveniente realizar un perfil de los trabajadores que asumen estas funciones para saber si disponen de la formación necesaria y si desarrollan su labor en estructuras adecuadas a sus habilidades. 
Por ello, el objeto de este estudio es analizar el perfil de los responsables de la gestión de la comunicación interna en las empresas que operan en España. En concreto, se persiguen los siguientes objetivos:

a) Las características socio-laborales de los profesionales de $\mathrm{Cl}$.

b) El posicionamiento, estructura y financiación de los equipos de $\mathrm{Cl}$ en las compañías.

c) Las relaciones de los equipos de $\mathrm{Cl}$ con los demás departamentos de la empresa.

Para la consecución de estos objetivos se tendrán en cuenta las diferencias en la gestión de la $\mathrm{Cl}$ según el tamaño de las empresas y su ámbito de implantación territorial.

De manera específica, la presente investigación trata de responder a las siguientes preguntas de investigación:

1. ¿De qué áreas del conocimiento proceden los responsables de $\mathrm{Cl}$ en España? ¿Han cursado formación especializada para el puesto que desempeñan?

2. ¿Cómo se organizan los departamentos responsables de la $\mathrm{Cl}$ en las empresas? ¿Qué perspectivas de futuro tienen?

3. ¿Cómo se integra la $\mathrm{Cl}$ en el funcionamiento de la empresa?

4. ¿Existen diferencias en los equipos responsables de la $\mathrm{Cl}$ de las empresas nacionales y multinacionales? ¿̇y entre los equipos de $\mathrm{Cl}$ de las empresas pequeñas, medianas y grandes?

\section{METODOLOGÍA}

\subsection{MUESTRA}

Para trazar los perfiles de los responsables de la $\mathrm{Cl}$ se han utilizado datos procedentes del Estudio sobre el Estado de la Comunicación Interna en España realizado por la asociación Dircom durante el primer trimestre de 2018. Para realizar el estudio se diseñó un cuestionario adaptado a partir del empleado previamente por ASCAI y FEIEA European Association of Internal Communication - (Nelli, 2018). El cuestionario contó 
con 37 preguntas y fue estructurado en diversos apartados que trataban los siguientes aspectos: el posicionamiento del equipo de $\mathrm{Cl}$; sus estrategias, objetivos y actividades; sus procesos de evaluación; el contexto empresarial; y las prioridades, problemas y retos a los que se enfrentan. El cuestionario se remitió vía online a los socios (1.022) y debía ser cumplimentado por los profesionales que tuvieran responsabilidades en la gestión de la $\mathrm{Cl}$. El tiempo aproximado para cumplimentarlo era de 20 minutos.

Así, se obtuvieron 118 encuestas a través de un muestreo no probabilístico por autoselección (Couper, 2000; Rodríguez y González, 2014). El 60.2\% de los participantes eran mujeres y el $39.8 \%$ hombres. En cuanto a la edad, el 1.7\% tenía menos de 25 años, el $4.2 \%$ tenía entre 26 y 30 , el $17 \%$ entre 31 y 40 , el $68.7 \%$ entre 41 y 50 , el $0.8 \%$ entre 51 y 60 y el $7.6 \%$ más de 60 años. Considerando la implantación territorial de la empresa en la que trabajaban, el $45.8 \%$ de trabajaba en una multinacional con sede en España, y el $54.2 \%$ en una nacional (concretamente, el 34.7\% en una empresa nacional con más de un centro de trabajo y el $19.5 \%$ en una nacional con un único centro de trabajo). Respecto al tamaño de las compañías, el $72 \%$ de los participantes ejercían en una gran empresa (más de 250 empleados), el 9.3\% en una mediana (entre 51 y 250 empleados), el $10.2 \%$ en una pequeña (entre 11 y 50 empleados) y el $8.5 \%$ en una microempresa (entre 1 y 10 empleados).

\subsection{ANÁLISIS DE DATOS}

Se ha realizado un análisis descriptivo de los ítems del cuestionario relativos a las características formativas y laborales de los profesionales de $\mathrm{Cl}$ y al posicionamiento de sus equipos en las empresas.

Para estudiar las diferencias en las evaluaciones de los participantes según la implantación territorial y tamaño de las empresas se ha utilizado el estadístico chicuadrado, la prueba $t$ de Student para muestras independientes y el ANOVA de un factor, en función del tipo de variable a analizar y el número de grupos de comparación.

El valor de significación establecido es <.05.

El análisis de los datos se ha realizado con el paquete estadístico SPSS, versión 21.0. 


\section{RESULTADOS}

\subsection{CARACTERÍSTICAS FORMATIVAS Y LABORALES DE LOS RESPONSABLES DE CI}

Todos los profesionales de $\mathrm{Cl}$ del estudio tienen titulación universitaria; la más habitual es la de periodismo (52.5\%), seguida de publicidad y relaciones públicas (22.9\%), económicas y empresariales (10.2\%) y derecho (6.8\%) (Gráfico 1). Hay un $8.4 \%$ de participantes que posee más de una titulación superior (el $5.1 \%$ tiene dos, el $2.5 \%$ tres y el $0.8 \%$ cinco) y un $10.6 \%$ que ha cursado titulaciones que, en principio, no guardan relación directa con el puesto de comunicación que desempeñan (historia del arte, dirección escénica y dramaturgia, ciencias de la educación, etc.).

Gráfico 1. Titulaciones académicas de los responsables de la gestión de $\mathrm{Cl}$ del estudio.

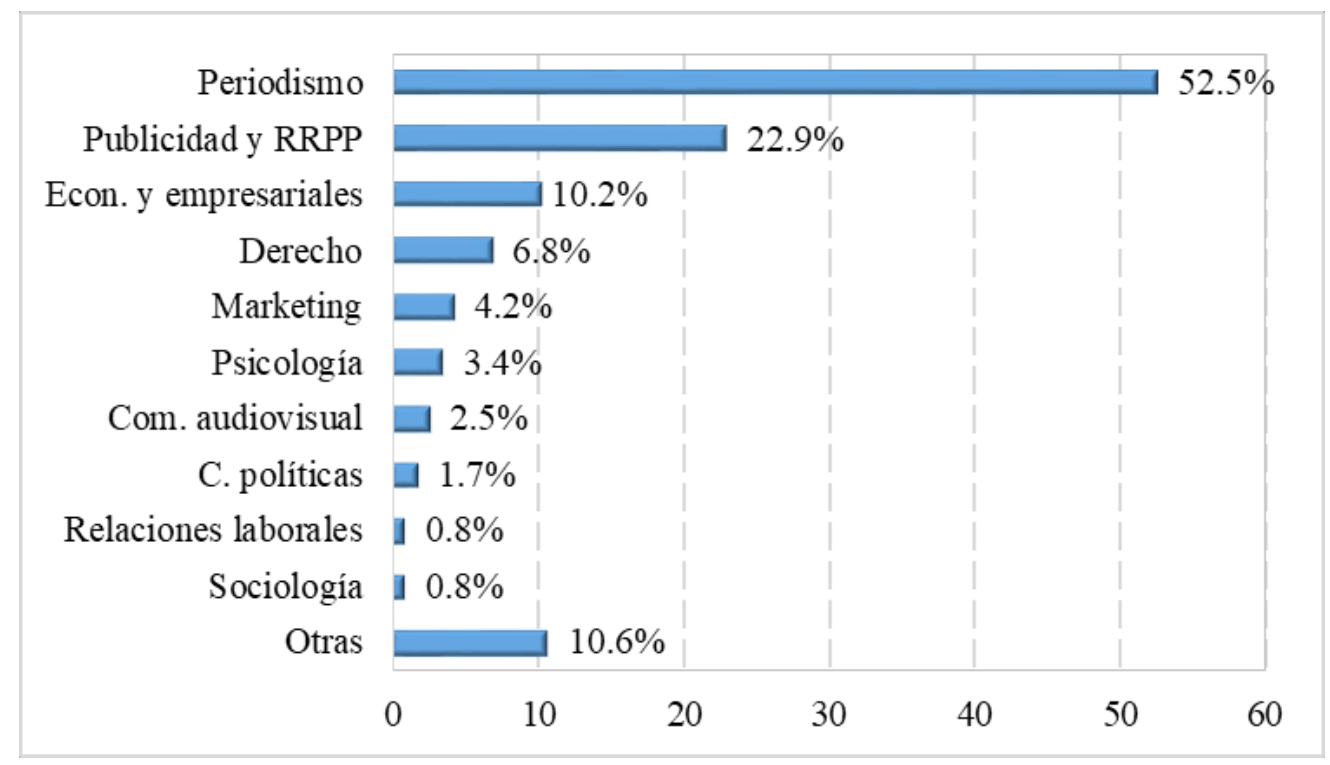

Fuente: elaboración propia.

La formación académica del $84.7 \%$ de los profesionales se completa con estudios de posgrado (máster o doctorado). En concreto, un $47.4 \%$ se ha especializado en comunicación corporativa, el 15.3\% en marketing y dirección comercial, el $8.5 \%$ en redes sociales, el $6.8 \%$ en MBA, el $6.8 \%$ en RRHH y el $5.1 \%$ en nuevas tecnologías. Además, un $17.8 \%$ de ellos posee varias titulaciones de posgrado.

Respecto al cargo que los responsables de $\mathrm{Cl}$ tienen asignado en la empresa, el más habitual es el de director de comunicación (51.7\%) (Gráfico 2); y un 11.9\% de ellos ocupa 
varias funciones dentro de su empresa (el $9.3 \%$ tiene dos puestos, el $1.7 \%$ tres y el $0.9 \%$ cuatro).

Gráfico 2. Puesto de los participantes en su empresa

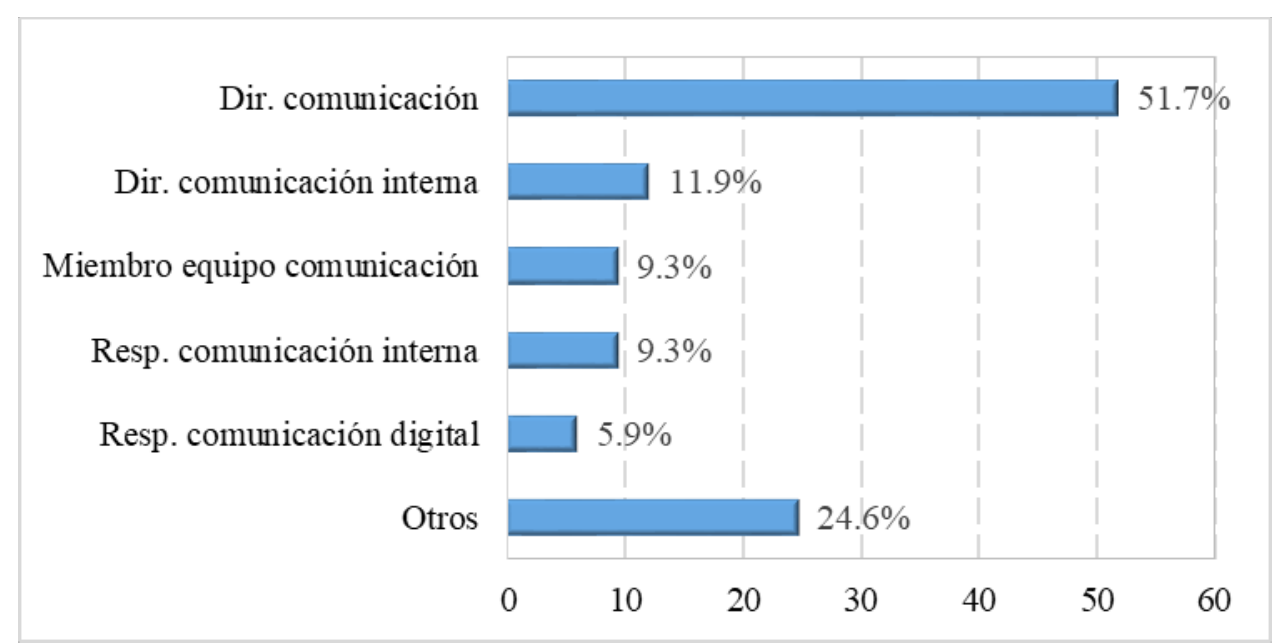

Fuente: elaboración propia.

\subsection{POSICIONAMIENTO DE LA Cl}

Analizando la organización de las empresas, el $85.6 \%$ de las estudiadas tienen una persona o un departamento concreto que se dedica a la $\mathrm{Cl}$. La cantidad de empresas que deciden delegar la $\mathrm{Cl}$ en un agente específico difiere significativamente según su implantación territorial $\left(\chi^{2}(1, N=118)=8.459 ; p=.012\right)$. Así, mientras que en las empresas nacionales lo hacen el 78.1\%, en las multinacionales hasta el $94.4 \%$ (Tabla 1 ).

Gráfico 3. Formas de gestión de la $\mathrm{Cl}$ en la empresa de los participantes.

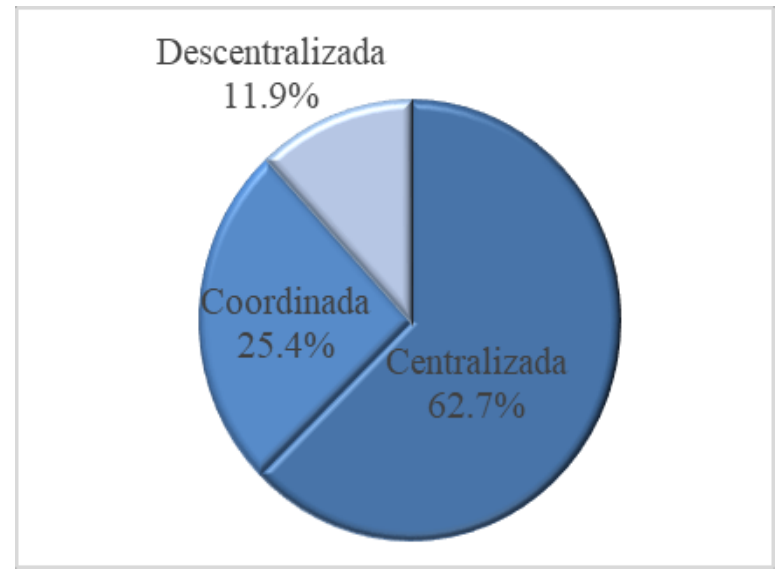

Fuente: elaboración propia.

RAEIC, Revista de la Asociación Española de Investigación de la Comunicación vol. 6, núm. 11 (2019), 99-118 
En cuanto a la gestión de la $\mathrm{Cl}$ (Gráfico 3), la que se realiza de forma centralizada es la más habitual (el equipo central de $\mathrm{Cl}$ define las estrategias y contenidos corporativos y los equipos locales las adaptan); la gestión coordinada (el equipo central de $\mathrm{Cl}$ actúa como punto de referencia de los equipos locales) la efectúan una de cada cuatro y la descentralizada (cada equipo local tiene su propio director de $\mathrm{Cl}$ que define la estrategia y los contenidos) una de cada diez.

Tabla 1. Posicionamiento del equipo de $\mathrm{Cl}$ según la implantación territorial y el tamaño de la empresa.

\begin{tabular}{|c|c|c|c|}
\hline \multicolumn{4}{|l|}{ Por su implantación territorial } \\
\hline & Nacionales & Multinacionales & Total empresas \\
\hline $\begin{array}{c}\text { La empresa tiene una persona o } \\
\text { departamento para la } \mathrm{Cl}\end{array}$ & $78.1 \%$ & $94.4 \%$ & $85.6 \%$ \\
\hline $\begin{array}{c}\text { Organización de la } \mathrm{Cl}: \\
\text { Centralizada } \\
\text { Coordinada } \\
\text { Descentralizada }\end{array}$ & $\begin{array}{c}71.9 \% \\
20.3 \% \\
7.8 \%\end{array}$ & $\begin{array}{l}51.8 \% \\
31.5 \% \\
16.7 \%\end{array}$ & $\begin{array}{l}62.7 \% \\
25.4 \% \\
11.9 \%\end{array}$ \\
\hline $\begin{array}{c}\text { Dpto. al que pertenece la } \mathrm{Cl} \text { : } \\
\text { RRHH } \\
\text { Comunicación corporativa } \\
\text { Otro }\end{array}$ & $\begin{array}{l}21.9 \% \\
40.6 \% \\
37.5 \% \\
\end{array}$ & $\begin{array}{l}14.8 \% \\
70.4 \% \\
14.8 \% \\
\end{array}$ & $\begin{array}{l}18.6 \% \\
54.2 \% \\
27.1 \% \\
\end{array}$ \\
\hline \multicolumn{4}{|l|}{ Por el tamaño de la empresa } \\
\hline & Micro y pequeñas & Medianas & Grandes \\
\hline $\begin{array}{c}\text { La empresa tiene una persona o } \\
\text { departamento para la } \mathrm{Cl}\end{array}$ & $63.6 \%$ & $90.9 \%$ & $90.6 \%$ \\
\hline $\begin{array}{c}\text { Organización de la } \mathrm{Cl}: \\
\text { Centralizada } \\
\text { Coordinada } \\
\text { Descentralizada }\end{array}$ & $\begin{array}{c}77.3 \% \\
18.2 \% \\
4.5 \%\end{array}$ & $\begin{array}{c}72.7 \% \\
27.3 \% \\
0.0 \%\end{array}$ & $\begin{array}{l}57.6 \% \\
27.1 \% \\
15.3 \%\end{array}$ \\
\hline $\begin{array}{c}\text { Dpto. al que pertenece la } \mathrm{Cl} \text { : } \\
\text { RRHH } \\
\text { Comunicación corporativa } \\
\text { Otro }\end{array}$ & $\begin{array}{l}22.7 \% \\
36.4 \% \\
40.9 \%\end{array}$ & $\begin{array}{c}9.1 \% \\
36.4 \% \\
54.5 \%\end{array}$ & $\begin{array}{l}18.8 \% \\
61.2 \% \\
20.0 \%\end{array}$ \\
\hline
\end{tabular}

Fuente: elaboración propia.

Los departamentos a los que se ha asignado el equipo de $\mathrm{Cl}$ son los siguientes: en el $54.3 \%$ de las empresas al de comunicación corporativa, en el $18.6 \%$ al de RRHH y en el $27.1 \%$ a otros departamentos (entre ellos, el 6\% a marketing, el 2.5\% a estrategias y desarrollo organizacional, y el $2.5 \%$ a gerencia). Considerando la implantación de la empresa, los departamentos a los que se adjudica la $\mathrm{Cl}$ difieren significativamente: en las multinacionales se asigna más al departamento de comunicación corporativa que en 
las empresas nacionales $(70.4 \%$ frente al $40.6 \%)\left(\chi^{2}(1, N=118)=11.119 ; p=.004\right)$. Según el tamaño de la empresa, las diferencias también son estadísticamente significativas $\left(\chi^{2}(2, N=118)=8.383 ; p=.015\right)$ entre pequeñas y medianas empresas respecto a las grandes (el $61.2 \%$ de estas últimas asignan la $\mathrm{Cl}$ al departamento de comunicación corporativa).

\subsection{ESTRUCTURA DE LOS EQUIPOS DE CI}

Examinando los trabajadores que componen el equipo de $\mathrm{Cl}$ en las empresas (Tabla 2), en el $35.6 \%$ de ellas están integrados por una única persona, en el $42.4 \%$ hay de dos a tres personas y en el $22 \%$ cuatro o más. Se han detectado diferencias significativas en el número de empleados que trabajan en $\mathrm{Cl}$ en las empresas nacionales y en las multinacionales $\left(\chi^{2}(2, N=118)=10.754 ; p=.005\right)$, contando estás últimas con más trabajadores.

Tabla 2. Estructura del equipo de $\mathrm{Cl}$ según la implantación territorial y el tamaño de la empresa.

\begin{tabular}{|c|c|c|c|}
\hline \multicolumn{4}{|l|}{ Por su implantación territorial } \\
\hline & Nacionales & Multinacionales & Total empresas \\
\hline $\begin{array}{l}\text { № de empleados que trabajan en } \mathrm{Cl} \text { : } \\
1 \text { empleado } \\
\text { De } 2 \text { a } 3 \text { empleados } \\
4 \text { y más empleados }\end{array}$ & $\begin{array}{l}48.4 \% \\
31.3 \% \\
20.3 \%\end{array}$ & $\begin{array}{l}20.3 \% \\
55.6 \% \\
24.1 \%\end{array}$ & $\begin{array}{l}35.6 \% \\
42.4 \% \\
22.0 \%\end{array}$ \\
\hline $\begin{array}{l}\text { Variación en los últimos } 3 \text { años del } \\
\text { equipo de } \mathrm{Cl} \text { : } \\
\text { Ha disminuido } \\
\text { Se ha quedado igual } \\
\text { Ha aumentado }\end{array}$ & $\begin{array}{l}14.1 \% \\
56.3 \% \\
29.7 \%\end{array}$ & $\begin{array}{l}5.6 \% \\
44.4 \% \\
50.0 \%\end{array}$ & $\begin{array}{l}10.2 \% \\
50.8 \% \\
39.0 \%\end{array}$ \\
\hline $\begin{array}{l}\text { Han incorporado a un experto en } \\
\text { comunicación digital }\end{array}$ & $38.3 \%$ & $35.9 \%$ & $37.2 \%$ \\
\hline $\begin{array}{l}\text { Planifican aumentar su equipo de } \mathrm{Cl} \text { en } \\
\text { los próximos } 3 \text { años }\end{array}$ & $28.1 \%$ & $40.7 \%$ & $33.9 \%$ \\
\hline $\begin{array}{l}\text { El dpto. de Cl cuenta con el apoyo de } \\
\text { consultoras externas }\end{array}$ & $37.5 \%$ & $40.7 \%$ & $39.0 \%$ \\
\hline \multicolumn{4}{|l|}{ Por el tamaño de la empresa } \\
\hline & $\begin{array}{l}\text { Micro y } \\
\text { pequeñas }\end{array}$ & Medianas & Grandes \\
\hline $\begin{array}{l}\text { № de empleados que trabajan en } \mathrm{Cl} \text { : } \\
1 \text { empleado } \\
\text { De } 2 \text { a } 3 \text { empleados } \\
4 \text { y más empleados }\end{array}$ & $\begin{array}{l}50.0 \% \\
36.4 \% \\
13.6 \%\end{array}$ & $\begin{array}{l}54.5 \% \\
36.4 \% \\
9.1 \%\end{array}$ & $\begin{array}{l}29.4 \% \\
44.7 \% \\
25.9 \%\end{array}$ \\
\hline
\end{tabular}

RAEIC, Revista de la Asociación Española de Investigación de la Comunicación vol. 6, núm. 11 (2019), 99-118 


\begin{tabular}{|c|c|c|c|}
\hline $\begin{array}{l}\text { Variación en los últimos } 3 \text { años del } \\
\text { equipo de } \mathrm{Cl} \text { : } \\
\text { Ha disminuido } \\
\text { Se ha quedado igual } \\
\text { Ha aumentado }\end{array}$ & $\begin{array}{l}4.5 \% \\
77.3 \% \\
18.2 \%\end{array}$ & $\begin{array}{l}9.1 \% \\
54.5 \% \\
36.4 \%\end{array}$ & $\begin{array}{l}11.8 \% \\
43.5 \% \\
44.7 \%\end{array}$ \\
\hline $\begin{array}{l}\text { Han incorporado a un experto en } \\
\text { comunicación digital }\end{array}$ & $41.2 \%$ & $50.0 \%$ & $34.9 \%$ \\
\hline $\begin{array}{l}\text { Planifican aumentar su equipo de } \mathrm{Cl} \text { en } \\
\text { los próximos } 3 \text { años }\end{array}$ & $18.2 \%$ & $36.4 \%$ & $37.6 \%$ \\
\hline $\begin{array}{l}\text { El dpto. de } \mathrm{Cl} \text { cuenta con el apoyo de } \\
\text { consultoras externas }\end{array}$ & $36.4 \%$ & $27.3 \%$ & $41.2 \%$ \\
\hline
\end{tabular}

Fuente: elaboración propia.

En el $50.8 \%$ de las empresas la plantilla de $\mathrm{Cl}$ ha permanecido estable en los últimos tres años; aunque considerando el tamaño de las empresas, las variaciones de plantilla en las pequeñas ha sido diferente al de las empresas medianas y grandes (que han registrado más crecimiento $)\left(\chi^{2}(2, \mathrm{~N}=118)=7.556 ; p=.023\right)$.

Además, el $37.2 \%$ de las empresas han incorporado a su plantilla a un experto en comunicación digital, el 33.9\% planean aumentar sus equipos de $\mathrm{Cl}$ en los próximos tres años y en un $39 \%$ el departamento de $\mathrm{Cl}$ suele contar en su trabajo con el apoyo de agencias y consultoras externas

\subsection{FINANCIACIÓN DE LA CI}

Considerando la financiación de los equipos de $\mathrm{Cl}$ en las empresas (Tabla 3), en el 58.5\% de las del estudio se asigna al departamento un presupuesto propio para gestionar su funcionamiento. La atribución de presupuesto específico al equipo de $\mathrm{Cl}$ es significativamente más habitual $\left(\chi^{2}(1, N=118)=9.978 ; p=.002\right)$ en las multinacionales (74.1\%) que en las empresas autóctonas (45.3\%) y también $\left(\chi^{2}(2, N=118)=7.118 ; p=\right.$ .028) en la empresas grandes (65.9\%) que en las medianas o pequeñas ( $45.5 \%$ y $36.4 \%$ ).

Tabla 3. Financiación de la $\mathrm{Cl}$, implantación territorial y tamaño de la empresa.

\begin{tabular}{|c|c|c|c|}
\hline \multicolumn{4}{|l|}{ Por su implantación territorial } \\
\hline & Nacionales & Multinacionales & Total empresas \\
\hline $\begin{array}{l}\text { El dpto. de } \mathrm{Cl} \text { tiene presupuesto } \\
\text { propio asignado }\end{array}$ & $45.3 \%$ & $74.1 \%$ & $58.5 \%$ \\
\hline $\begin{array}{l}\text { En el último año su presupuesto: } \\
\text { Ha incrementado } \\
\text { Se ha quedado igual }\end{array}$ & $\begin{array}{l}21.7 \% \\
68.3 \%\end{array}$ & $\begin{array}{l}37.3 \% \\
54.9 \%\end{array}$ & $\begin{array}{l}28.8 \% \\
62.2 \%\end{array}$ \\
\hline
\end{tabular}

RAEIC, Revista de la Asociación Española de Investigación de la Comunicación vol. 6, núm. 11 (2019), 99-118 


\begin{tabular}{|c|c|c|c|}
\hline Ha disminuido & $10.0 \%$ & $7.8 \%$ & $9.0 \%$ \\
\hline $\begin{array}{l}\text { Previsión de la variación de su } \\
\text { presupuesto para el próximo año: } \\
\text { Disminuirá } \\
\text { Se mantendrá igual } \\
\text { Aumentará } \\
\text { No está seguro }\end{array}$ & $\begin{array}{l}0.0 \% \\
62.5 \% \\
25.0 \% \\
12.5 \%\end{array}$ & $\begin{array}{l}3.7 \% \\
66.7 \% \\
24.1 \% \\
5.6 \%\end{array}$ & $\begin{array}{l}1.7 \% \\
64.4 \% \\
24.6 \% \\
9.3 \%\end{array}$ \\
\hline \multicolumn{4}{|l|}{ Por el tamaño de la empresa } \\
\hline & Micro y pequeñas & Medianas & Grandes \\
\hline $\begin{array}{l}\text { El dpto. de } \mathrm{Cl} \text { tiene presupuesto } \\
\text { propio asignado }\end{array}$ & $36.4 \%$ & $45.5 \%$ & $65.9 \%$ \\
\hline $\begin{array}{l}\text { En el último año su presupuesto } \\
\text { Ha incrementado } \\
\text { Se ha quedado igual } \\
\text { Ha disminuido }\end{array}$ & $\begin{array}{l}23.8 \% \\
71.4 \% \\
4.8 \%\end{array}$ & $\begin{array}{l}20.0 \% \\
80.0 \% \\
0.0 \%\end{array}$ & $\begin{array}{l}31.3 \% \\
57.5 \% \\
11.3 \%\end{array}$ \\
\hline $\begin{array}{l}\text { Previsión de la variación de su } \\
\text { presupuesto para el próximo año } \\
\text { Disminuirá } \\
\text { Se mantendrá igual } \\
\text { Aumentará } \\
\text { No está seguro }\end{array}$ & $\begin{array}{l}0.0 \% \\
59.1 \% \\
31.8 \% \\
9.1 \%\end{array}$ & $\begin{array}{l}0.0 \% \\
63.6 \% \\
27.3 \% \\
9.1 \%\end{array}$ & $\begin{array}{l}2.4 \% \\
65.9 \% \\
22.4 \% \\
9.4 \%\end{array}$ \\
\hline
\end{tabular}

Fuente: elaboración propia.

La asignación presupuestaria del último año a la $\mathrm{Cl}$ se ha mantenido estable en el $62.2 \%$ de las empresas y en el $64.4 \%$ de ellas sus responsables creen que en el ejercicio próximo también se mantendrá igual.

\subsection{RELACIONES DEL EQUIPO DE CI CON LOS DEMÁS DEPARTAMENTOS DE LA EMPRESA}

El gráfico 4 muestra que los equipos de $\mathrm{Cl}$ están involucrados con todos los departamentos de las empresas, pero hay que señalar que, aunque la $\mathrm{Cl}$ no está aislada de ninguno de los demás departamentos ya que la evaluación de la relación de cercanía que mantienen con ellos según sus responsables no es desdeñable en ningún caso, sí que se observa que con algunos equipos la relación es más intensa. Se ha señalado que los departamentos de la empresa con los que la $\mathrm{Cl}$ mantiene una relación más cercana son el de comunicación externa y el equipo directivo (evaluadas, respectivamente, con una media de 4.53 y 4.37 sobre un máximo de 5). Por el contrario, con el departamento legal, el financiero y el de auditoría son con los que la relación es menor (de 2.94, 2.81 y 2.44 de media, respectivamente). 
Gráfico 4. Valores medios de la evaluación de la cercanía que mantiene el equipo de $\mathrm{Cl}$ con los demás departamentos de la empresa*.

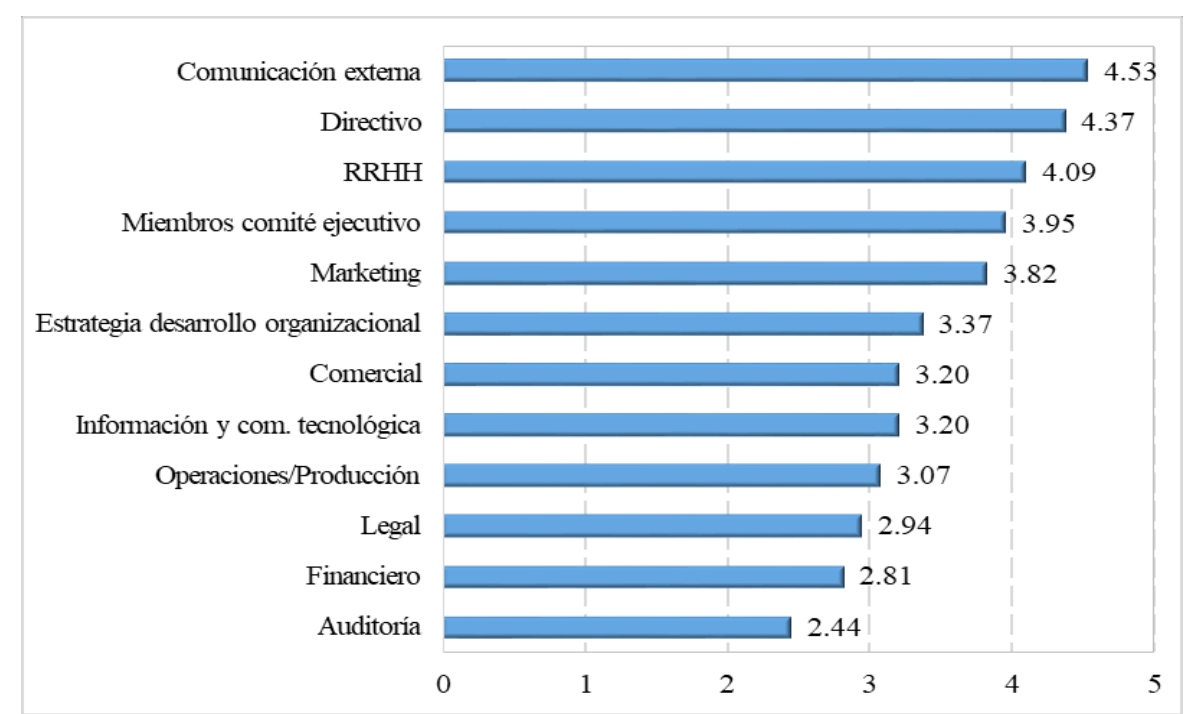

Fuente: elaboración propia.

*NOTA: 1 = poco cercana a 5 = muy cercana

Gráfico 5. Valores medios de la evaluación de la cercanía que mantiene el equipo de $\mathrm{Cl}$ con los demás departamentos según la implantación territorial de la empresa*.

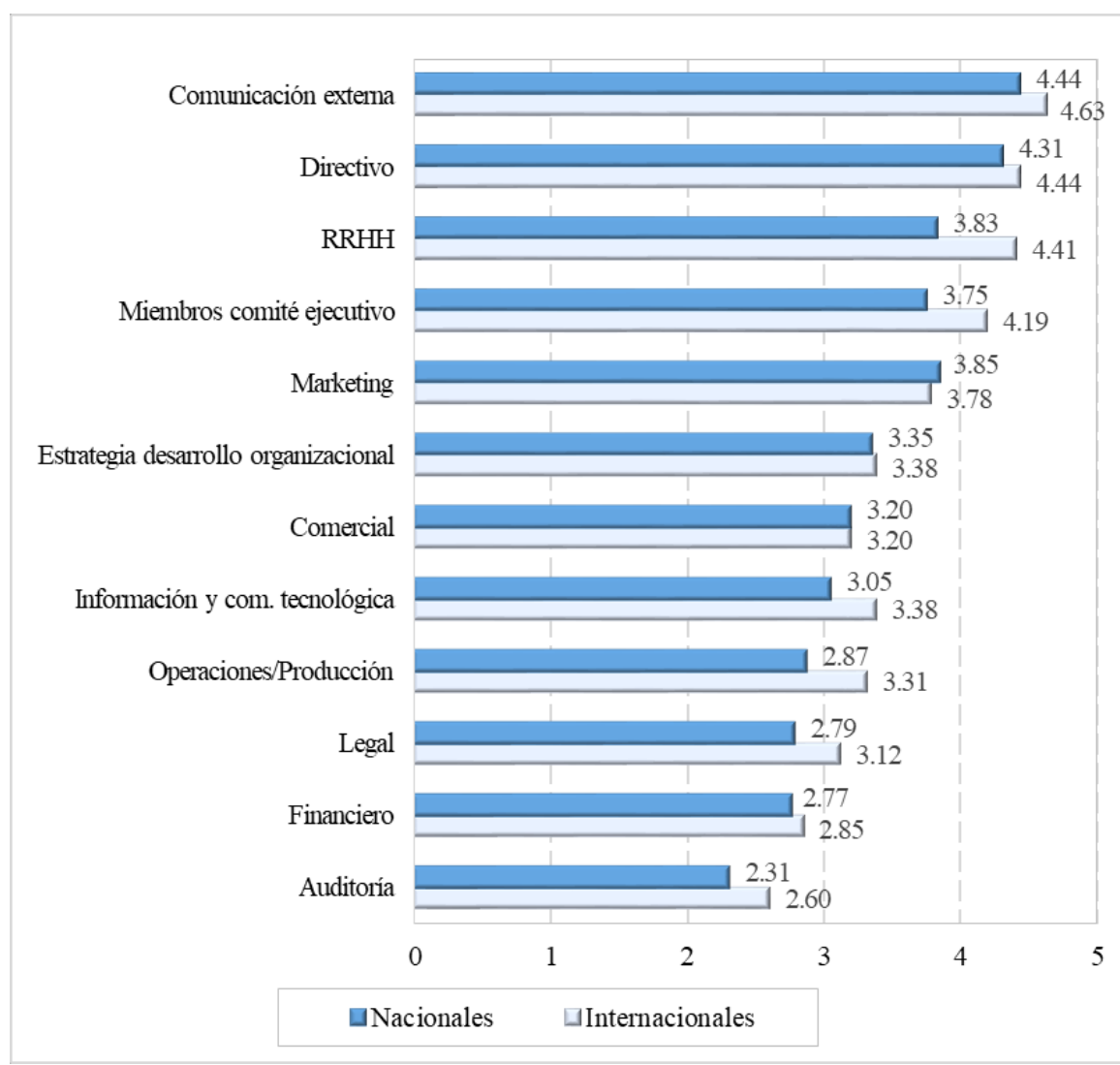

RAEIC, Revista de la Asociación Española de Investigación de la Comunicación vol. 6, núm. 11 (2019), 99-118 
Fuente: elaboración propia.

*NOTA: 1 = poco cercana a 5 = muy cercana

Si se tiene en cuenta la implantación territorial de las empresas, los análisis muestran que la cercanía del equipo de $\mathrm{Cl}$ con los miembros del comité ejecutivo es mayor en las empresas multinacionales (4.19) que en las nacionales (3.75), siendo las diferencias estadísticamente significativas $(t(108.704)=-2.17 ; p=.033)$. Asimismo, también es significativamente superior $(t(112.947)=-2.79 ; p=.006)$ la relación que se registra en las multinacionales con el departamento de RRHH (4.19 frente a 3.75) (Gráfico 5).

Considerando el tamaño de las empresas, en las evaluaciones de los responsables de $\mathrm{Cl}$ de la proximidad que mantiene su equipo con los demás departamentos los ANOVA no presentan diferencias estadísticamente significativas (Gráfico 6).

Gráfico 6. Valores medios de la evaluación de la cercanía que mantiene el equipo de $\mathrm{Cl}$ con los demás departamentos según el tamaño de la empresa*.

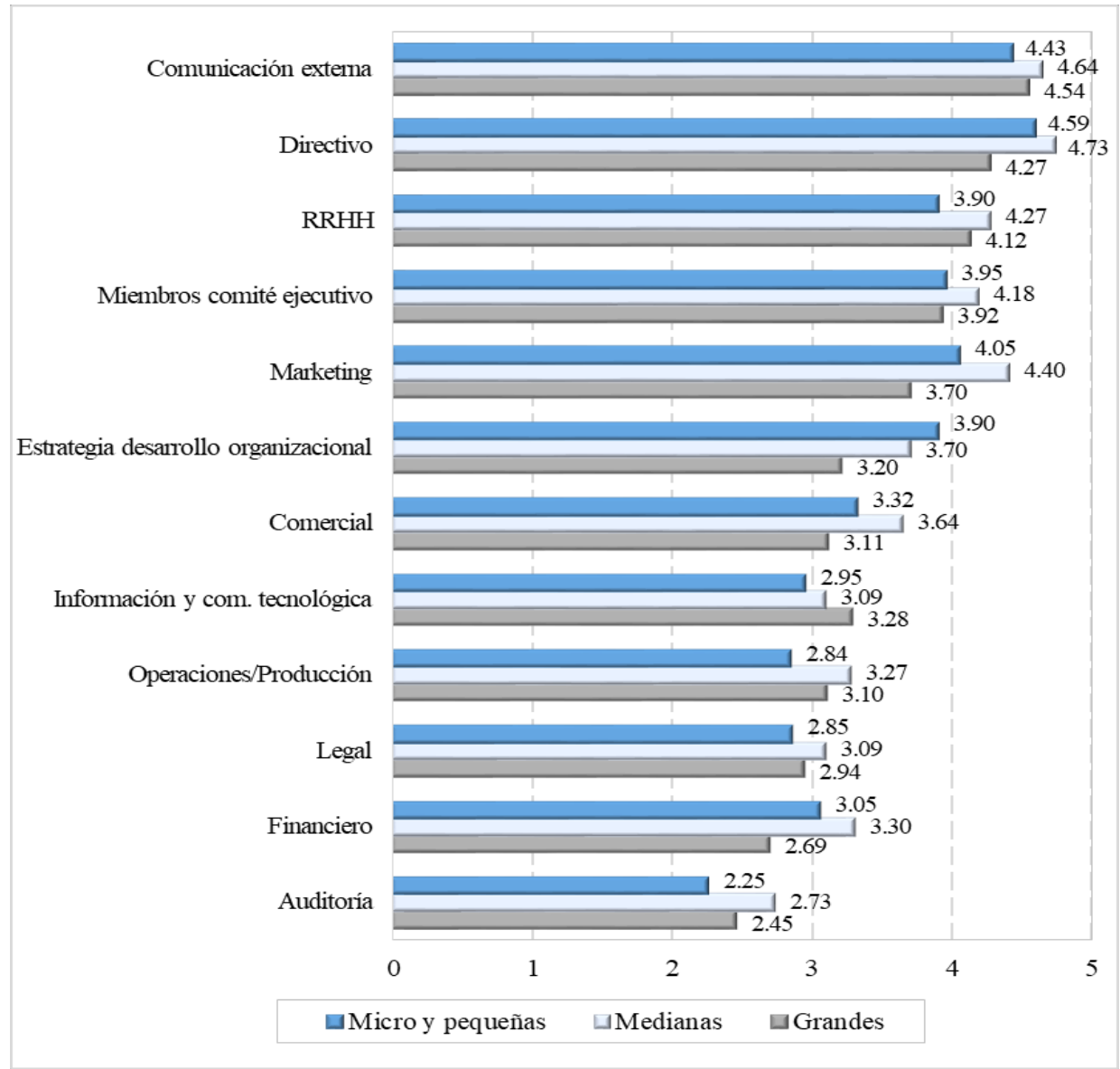

Fuente: elaboración propia.

*NOTA: 1 = poco cercana a 5 = muy cercana

RAEIC, Revista de la Asociación Española de Investigación de la Comunicación vol. 6, núm. 11 (2019), 99-118 


\section{CONCLUSIONES Y DISCUSIÓN}

Los responsables de la gestión de la $\mathrm{Cl}$ en las empresas que han participado en el estudio son profesionales formados académicamente en la rama de las ciencias sociales, principalmente del periodismo. Son trabajadores altamente cualificados que suelen contar (el 84.7\%) con estudios de posgrado. Muchos de ellos suelen tener atribuido el cargo de director de comunicación en general, y solo en el $17.8 \%$ son designados específicamente como directores o responsables de $\mathrm{Cl}$, a pesar de que nueve de cada diez tengan una persona o un equipo específico para realizar estas actividades.

Como muestran estudios previos (Nelli, 2018), la forma de gestión de la Cl más extendida es la centralizada (el equipo central de $\mathrm{Cl}$ define las estrategias y contenidos corporativos y los equipos locales las adaptan), estando asignados sus equipos en más de la mitad de las empresas a los departamentos de comunicación corporativa, un dato muy similar al arrojado en investigaciones anteriores (Estudio de Comunicación, 2014). Menos habitual es que la $\mathrm{Cl}$ se integre en el departamento de $\mathrm{RRHH}(18.6 \%)$ o de marketing (6\%).

La existencia de un departamento exclusivamente dedicado a la comunicación interna depende del tamaño y del tipo de empresa, y es más habitual en las compañías grandes y multinacionales, según el estudio "Internal communication in Europe", llevado a cabo por ASCAI y FEIEA (Nelli, 2018). Según este estudio, casi el 60\% de las empresas cuenta con un departamento dedicado a la comunicación interna.

Los equipos de $\mathrm{Cl}$ actuales suelen estar formados por pocos trabajadores; solo el $22 \%$ de ellos tienen cuatro o más componentes, a pesar de que en los tres últimos años en cuatro de cada diez empresas hayan aumentado los trabajadores que los integran, sobre todo incorporando expertos en comunicación digital. Los equipos presentes necesitan en muchos casos contar con el apoyo de consultoras externas aunque, a medio plazo, planifican seguir creciendo, una tendencia de crecimiento que también señalan estudios previos (Nelli, 2018). 
En cuanto a la financiación de los equipos de $\mathrm{Cl}$, seis de cada diez cuentan con un presupuesto propio que tiene bastante estabilidad a corto plazo.

Los equipos de $\mathrm{Cl}$ de las empresas están integrados en la estructura empresarial ya que están relacionados con todos los departamentos. Sin embargo, sí que se ha detectado que con los equipos de comunicación externa, el directivo, el de RRHH y los miembros del comité ejecutivo la relación es más intensa, en línea con los resultados del estudio de ASCAI y FEIEA a nivel europeo (Nelli, 2018).

Si se tiene en cuenta la implantación geográfica de las empresas, casi la totalidad de las multinacionales (94.4\%) tiene un equipo específico de $\mathrm{Cl}$ que, en mayor medida que en las nacionales, está integrado en el departamento de comunicación corporativa, tiene más trabajadores y se le asigna presupuesto específico. Aunque los departamentos de la empresa con los que los equipos de $\mathrm{Cl}$ de las entidades nacionales y multinacionales establecen lazos de relación más estrechos son los mismos, los equipos de las multinacionales tienen una relación más intensa que los de las nacionales con los miembros del comité ejecutivo y con los de RRHH.

Considerando el tamaño de las empresas, las grandes se integran en el departamento de comunicación corporativa y suelen tener presupuesto propio en mayor proporción que las pequeñas y medianas; y las medianas y grandes han registrado mayor crecimiento en los últimos tres años en sus plantillas de $\mathrm{Cl}$ que las pequeñas.

En definitiva, podemos concluir que los equipos de $\mathrm{Cl}$ en España están dirigidos por profesionales de alta cualificación y van ganando identidad propia dentro del esquema organizativo de las empresas, aunque parece que, en este proceso, van algo más adelantadas las empresas multinacionales y las de gran tamaño.

Precisamente en estas últimas es donde la cercanía entre el equipo de $\mathrm{Cl}$ y la alta dirección es mayor, según muestra el estudio. No obstante, todavía se puede mejorar mucho en este sentido. La relación de $\mathrm{Cl}$ con la dirección de la empresa sigue perfilándose como un tema crítico no solo para que la $\mathrm{Cl}$ se perciba como una función estratégica dentro de las organizaciones sino también para aumentar su eficacia y su 
calidad (Ingelmo et al., 2018). Un aspecto en el que convendría seguir ahondando en futuros estudios sobre la materia.

Este estudio ofrece una radiografía del estado actual de la comunicación interna en España sin precedentes hasta el momento. Sin embargo, tiene ciertas limitaciones que conviene tener en cuenta y que pueden abrir la puerta a futuras investigaciones. Aunque la muestra es representativa, sería interesante contar con una muestra más amplia y poder contrastar los datos cuantitativos con datos cualitativos procedentes de entrevistas en profundidad a profesionales de la comunicación interna y CEOs. La realización de un estudio de estas características de forma periódica permitiría, además, realizar un análisis comparativo y valorar la evolución de la función de $\mathrm{Cl}$ en las organizaciones.

\section{REFERENCIAS BIBLIOGRÁFICAS}

CIPR Inside (2017). Making it Count. Ciprinside.co.uk. Recuperado de http://www.ciprinside.co.uk/making-count-infographic/

Cooper, C. (2000). Web surveys: The questionnaire Design Challenge. En Proceedings of the 53rd session of the ISI. Recuperado de

http://isi.cbs.nl/iamamember/CD2/pdf/263.pdf

Couper, M. P. (2001). Web surveys: The questionnaire design challenge. Recuperado de

http://citeseerx.ist.psu.edu/viewdoc/download?doi=10.1.1.99.3436\&rep=rep1\&type= pdf

Corporate Excellence - Centre for Reputation Leadership (2018). Claves para entender la relación estratégica entre las áreas de Comunicación y Recursos Humanos. HR \& Dircom International Forum. Madrid. Recuperado de http://www.corporateexcellence.org/index.php/Compartimos-conocimiento/HRDircom-Claves-para-entender-la-relacion-estrategica-entre-las-areas-deComunicacion-y-Recursos-Humanos 
Cuenca, J. y Verazzi, L. (2018). Guía fundamental de la comunicación interna.

Barcelona: Editorial UOC.

Dircom (2018). Estado de la comunicación en España 2018. Dircom.org. Recuperado de http://www.dircom.org/actualidad-dircom/item/9176-estado-de-la-comunicacion-enespana-2018

Estudio de Comunicación (2014). La comunicación interna de las empresas cotizadas (vista por sus trabajadores). Estudiodecomunicacion.com. Recuperado de https://www.estudiodecomunicacion.com/servicios/la- comunicacion-interna-de-lasempresas-cotizadas-vista-por-sus- trabajadores/

IC Kollectif (2017). Disrupting the Function of IC. A Global Perspective. Recuperado de https://www.ickollectif.com/ebook.

Ingelmo, M., Navarro, C., y Sanz, J. Á. (2018). Determining factors of success in internal communication management in Spanish companies: The influence of social media. Corporate Communications: An International Journal, 23(3), 405-422. DOI: http://doi.org/10.1108/CCIJ-03-2017-0021.

Maier, C.D. y Andersen, M. A. (2017). Strategic internal communication of corporate heritage identity in a hypermodal context. Corporate Communications: An International Journal, 22, 36-59.

Men, R. L., y Bowen, S. A. (2016). Excellence in internal communication management. New York: Business Expert Press

Nelli, R. P. (2018). In Europe Communication. Ascai.it. Recuperado de http://www.ascai.it/uploads/files/RAPPORTI\%20ASCAI/E BOOK\%20IC\%20EUROPEAN \%20REPORT\%202018.pdf

Rodríguez, M. J. y González, M. J. (2014). Las encuestas autoadministradas por internet. Un estudio de caso: "las familias adoptivas y sus estilos de vida". Empíria, Revista de Metodología de Ciencias Sociales, 29, 155-175. 
Tench R., y Moreno, A. (2015). Mapping communication competencies for European practitioners: ECOPSI an EU study. Journal of Communication Management, 19, 39-61.

Tench R., Verčič D., Zerfass A., Moreno Á., Verhoeven P. (2017). Communication Excellence. How to develop, manage and lead exceptional communications. London: Palgrave Macmillan.

Tkalac Verčič, A., y Pološki Vokić, N. (2017). Engaging employees through internal communication. Public Relations Review, 43(5), pp. 885-893.

Verčič D., Zerfass A. (2016). A comparative excellence framework for communication management. Journal of Communication Management, 20, 270-288.

VMA Group (2018). The View. Recuperado de: http://www.vmagroup.com/the-view/ Volk, S. C., Berger, K., Zerfass, A., Bisswanger L., Fetzer M. y Köhler K. (2017). How to play the game. Strategic tools for managing corporate communications and creating value for your organization. Leipzig: Academic Society for Management \& Communication.

Xifra, J. (2014) Manual de relaciones públicas e institucionales. Segunda edición revisada y ampliada. Madrid: Editorial Tecnos.

Zerfass A., Franke, N. (2013). Enabling, Advising, Supporting, Executing: A theoretical framework for internal communication consulting within organizations. International Journal of Strategic Communication, 7, 118-135.

Zerfass, A., Moreno, A., Tench, R., Verčič, D. y Verhoeven, P. (2017). European Communication Monitor 2017. How strategic communication deals with the challenges of visualisation, social bots and hypermodernity. Results of a survey in 50 Countries. Brussels: EACD/ EUPRERA, QuadrigaMedia Berlin. Recuperado de https://de.slideshare.net/communicationmonitor/european-communication-monitor$\underline{2017}$. 
Zerfass, A., Verčič, D., Volk, S. C. (2017). Communication evaluation and measurement: Skills, practices and utilization in European organizations. Corporate Communications: An International Journal, 22, 2-18. 